\title{
Realization of Unidirectional Planar GaAs Nanowires on GaAs (110) Substrates
}

\author{
Ryan Dowdy, Donald A. Walko, Seth A. Fortuna, and Xiuling Li, Senior Member, IEEE
}

\begin{abstract}
A self-aligned unidirectional planar GaAs nanowire (NW) array is realized by growing on (110) GaAs substrates through the Au-catalyzed vapor-liquid-solid mechanism. All NWs on (110) substrates propagate along the [00-1] direction, yielding planar NWs with trapezoidal cross sections where the top surface and sidewalls are identified by micro $\mathrm{X}$-ray diffraction analysis to be [110], [010], and [100] facets, respectively. Depletion-mode long-channel metal-semiconductor field-effect transistors using these [00-1] GaAs NWs as channels exhibit well-defined dc output and transfer characteristics, confirming the high material quality of the NWs. Completely ordered site controlled arrays of planar NWs are demonstrated by growing on (110) substrates with Au catalyst nanoparticles patterned using electron beam lithography.
\end{abstract}

Index Terms-GaAs, metal-semiconductor field-effect transistor (MESFET), nanowire (NW), nanowire array.

\section{INTRODUCTION}

$\mathbf{S}$ EMICONDUCTOR nanowires (NWs) are becoming core nanotechnology building blocks for next-generation electronics [1], optoelectronics [2], nanoelectromechanical systems [3], and biological applications [4]. The ability to create ordered NW structures with controllability in size, orientation, position, and alignment is critical to future manufacturability and compatibility with existing semiconductor fabrication processes [5]. Growth via vapor-liquid-solid (VLS) mechanism is arguably the most commonly used method for the formation of single crystalline semiconductor NW structures including heterojunctions and p-n junctions in axial and radial directions.

However, most VLS NWs are grown vertically or out of plane with respect to their substrates. NWs grown in this fashion are prone to planar defects such as stacking faults and polytypic growth [6], which are problematic to device performance [7]. The out-of-plane orientation is also incompatible with traditional planar processes that are used in modern

Manuscript received January 9, 2012; accepted January 21, 2012. Date of publication March 7, 2012; date of current version March 23, 2012. This work was supported in part by Defense Advanced Research Projects Agency Young Faculty Award under Grant N66001-09-1-2107, Office of Naval Research Young Investigator Program YIP Award N000141110634, National Science Foundation Awards 1001928 (ECCS) and 1006581 (DMR), and by the Robert Rathbun Wilson Graduate Fellowship from Argonne National Laboratory (RD). Use of the Advanced Photon Source was supported by the U.S. Department of Energy, Office of Science, Office of Basic Energy Sciences, under Contract DE-AC02-06CH11357. The review of this letter was arranged by Editor G. Meneghesso.

R. Dowdy, S. A. Fortuna, and X. Li are with the Department of Electrical and Computer Engineering, University of Illinois, Urbana, IL 61801 USA (e-mail: rdowdy2@illinois.edu; seth.a.fortuna@gmail.com; xiuling@illinois.edu).

D. A. Walko is with the Advanced Photon Sources, Argonne National Laboratory, Argonne, IL 60439 USA (e-mail: d-walko@anl.gov).

Color versions of one or more of the figures in this letter are available online at http://ieeexplore.ieee.org.

Digital Object Identifier 10.1109/LED.2012.2186115
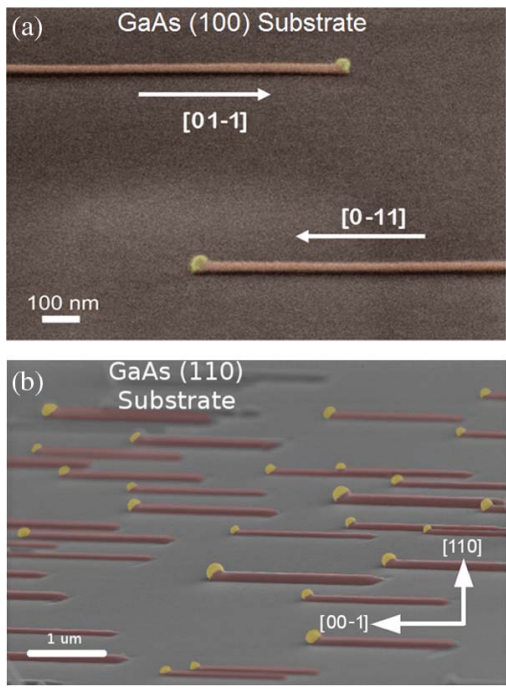

Fig. 1. SEM images $\left(75^{\circ}\right.$ tilted and false colored $)$ of self-aligned bidirectional and unidirectional planar NWs with the Au nanoparticles at the growth front. (a) Grown on a (100) substrate, with the planar NWs propagating randomly in either the [01-1] or the [0-11] direction as indicated. Adapted with permission from [9]. Copyright 2008 American Chemical Society. (b) Grown on a (110) substrate, with the planar NWs propagating unanimously in one single direction, [00-1].

electronics. NWs usually have to be removed from the surface through external force and transferred to a new host substrate where the final device is fabricated [8]. Recently, our research group has reported a controlled method to grow Au-catalyzed VLS GaAs NWs in a planar orientation $\langle 110\rangle$ epitaxially on GaAs (100) substrates [9], which are self-aligned and free of twin plane defects. Planar NW growth has also been reported on GaAs (311) substrates as well [10]. High-performance metal-semiconductor field-effect transistors (MESFETs) and high-electron-mobility transistors (HEMTs) have been demonstrated using these planar $\langle 110\rangle$ GaAs NWs as the channels [11], [12]. However, on (100) substrates, self-aligned planar NWs propagate in either [01-1] or [0-11] direction, i.e. parallel or antiparallel as shown in Fig. 1(a) [9]. This is because these two orientations are crystallographically equivalent, which clearly prevents complete alignment through site control of the Au catalysts. The purpose of this work is to eliminate the antiparallel propagation direction and achieve completely ordered NW arrays as grown. We demonstrate that the choice of substrate orientation allows direct control of the available planar growth directions. We report the growth of unidirectional planar NWs, structural characterization, and MESFET device results using these NWs as the channel. 
(a)

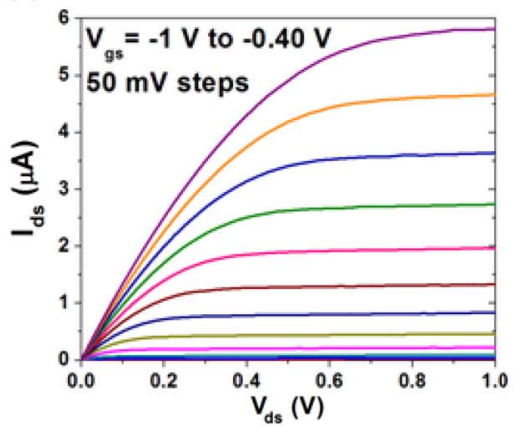

(b)

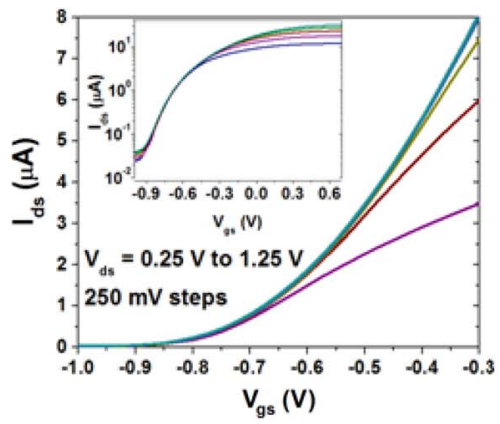

(c)

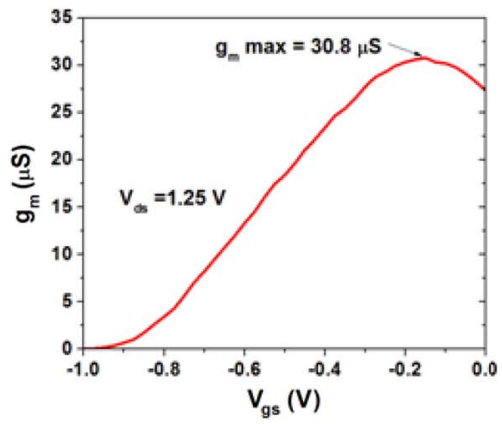

Fig. 2. DC output and transfer characteristics of a depletion-mode NW-MESFET using a [00-1] GaAs NW channel on a (110) GaAs substrate. (a) $I_{\mathrm{ds}}$ versus $V_{\mathrm{ds}}$ family of curves for $V_{\mathrm{gs}}$ from -1 to $-0.4 \mathrm{~V}$ with $50-\mathrm{mV}$ steps. (b) $I_{\mathrm{ds}}$ versus $V_{\mathrm{gs}}$ transfer characteristics for $V_{\mathrm{ds}}=0.25$ to $1.25 \mathrm{~V}$ with 250 -mV steps. The inset is $I_{\mathrm{ds}}$ versus $V_{\mathrm{gs}}$ on a semilog plot. (c) Transconductance $g_{m}$ versus $V_{\mathrm{gs}}$ plot with maximum $g_{m}$ of $30.8 \mu \mathrm{S}$ ( $75.9 \mathrm{mS} / \mathrm{mm}$ when normalized to the NW base width).

\section{EXPERIMENTAL}

VLS planar GaAs NWs were grown using 250-nm colloidal gold $(\mathrm{Au})$ nanoparticles as catalysts, and the growth was carried out in a horizontal-flow Aixtron metal organic chemical vapor deposition (MOCVD) reactor under atmospheric pressure. Epiready semi-insulating GaAs substrates of various orientations, including (100) and (110), were used for growth. For the growth of site-controlled NW arrays, a JEOL JBX-6000FS electron beam lithography system was used to pattern the GaAs substrates using PMMA resist, followed by the deposition of approximately $20 \mathrm{~nm}$ of Au thin film using an electron beam evaporator and then liftoff. To remove residual PMMA, the samples were exposed for $10 \mathrm{~min}$ to a $300-\mathrm{W} \mathrm{O}_{2}$ plasma and then soaked in three different solvent baths consisting of acetone, methanol, and isopropanol for $10 \mathrm{~min}$ each. Arsine $\left(\mathrm{AsH}_{3}\right)$, trimethylgallium (TMGa), and disilane $\left(\mathrm{Si}_{2} \mathrm{H}_{6}\right)$ were used as the arsenic (As), gallium ( $\mathrm{Ga})$, and silicon ( $\mathrm{Si}$, dopant) precursors, respectively. The desorption temperature in the MOCVD chamber was $625^{\circ} \mathrm{C}$, and the NW growth temperature was around $480^{\circ} \mathrm{C}$ with a growth rate of $\sim 57 \mathrm{~nm} / \mathrm{s}$. MESFETs were fabricated using $\mathrm{Ge} / \mathrm{Au} / \mathrm{Ni}$ for the source and drain ohmic contact and Ti/Au for the top gate Schottky contact. The sourceto-drain distance is $\sim 7 \mu \mathrm{m}$, and the gate length is $2 \mu \mathrm{m}$. More details of the device fabrication can be found elsewhere [11]. Postgrowth characterization was done with a Hitachi S-4800 field emission scanning electron microscope (SEM). Micro $\mathrm{X}$-ray diffraction experiments using $10-\mathrm{keV}$ synchrotron radiation were performed at the Sector 7 beamline of the Advanced Photon Source at Argonne National Laboratory. The X-ray beam was focused to an $\sim 20-\mu \mathrm{m}$ spot size, with measurements performed in grazing incidence geometry to enhance surface sensitivity.

\section{RESUltS AND Discussion}

In typical out-of-plane epitaxial growth on GaAs (100) substrates, GaAs NWs have been shown to grow in the $\langle 111\rangle B$ directions [13]. The NWs have equal chances to grow in either of the two $\langle 111\rangle B$ directions: $\left[\begin{array}{lll}1 & 1 & -1\end{array}\right]$ and $\left[\begin{array}{lll}1 & -1 & 1\end{array}\right]$. Under planar growth conditions on GaAs (100) substrates, GaAs NWs have equal chances to grow in the $\left[\begin{array}{lll}0 & 1 & -1\end{array}\right]$ or $\left[\begin{array}{lll}0 & -1 & 1\end{array}\right]$ crystal directions, parallel or antiparallel to each other [9], as shown in Fig. 1(a).

We have found that planar NWs tend to grow along the projections of $\langle 111\rangle B$ crystal directions onto the substrate plane. Since there is only one $\langle 111\rangle B$ direction on a (110) substrate, we are able to realize unidirectional planar NW growth using (110) substrates, as shown in Fig. 1(b). Through X-ray crystal truncation rod analysis of the facets' rods which originate at the 111 Bragg peak, the planar NWs are confirmed to grow unstrained and in full registry with the substrate in the [00-1] direction, with (100) and (010) side facets, i.e., a trapezoidal cross section with the (110) top facet parallel to the substrate surface and two $\langle 010\rangle$ facets that form a $45^{\circ}$ angle with the surface. The realization of completely parallel unidirectional planar NW growth has significant implications since it is now possible to make fully aligned arrays of NWs as will be demonstrated later.

Previously, we have reported device results of MESFETs [11] and HEMTs [12] using planar GaAs $\langle 110\rangle$ NWs on semi-insulating GaAs (100) substrates as the channels. Here, we demonstrate MESFETs utilizing [00-1] planar NWs on a GaAs (110) substrate to prove their viability as a FET device channel. Fig. 2 shows the dc characteristics of a depletionmode MESFET with an n-type Si-doped [00-1] planar NW as the channel. The family of $I_{\mathrm{ds}}-V_{\mathrm{gs}}$ curves exhibits a linear region, onset of pinchoff, and saturation as shown in Fig. 2(a), indicating well-defined channel modulation behavior. The transfer characteristics $\left(I_{\mathrm{ds}}-V_{\mathrm{gs}}\right)$ in Fig. 2(b) is shown for $V_{\mathrm{ds}}$ values in the range of 0.25 to $1.25 \mathrm{~V}$, with the semilog plot of the same type shown in the inset. The $I_{\mathrm{on} / \mathrm{off}}$ ratio of the device is $\sim 850$. Further increase of $I_{\text {on/off }}$ is expected with improved device isolation and reduction of parasitic deposition on the substrate. The subthreshold slope is $169 \mathrm{mV} / \mathrm{dec}$, and the threshold voltage is $-0.88 \mathrm{~V}$ taken at a $V_{\mathrm{ds}}$ of $100 \mathrm{mV}$. The maximum drive current $I_{\mathrm{ds}}-\max$ at a $V_{\mathrm{gs}}$ of $0.5 \mathrm{~V}$ is $96.3 \mu \mathrm{A} / \mu \mathrm{m}$; the transconductance $g_{m}$ is shown in Fig. 2(c) with maximum $g_{m}$ at $30.8 \mu \mathrm{S}$ or $75.9 \mathrm{mS} / \mathrm{mm}$, where the base width of the NW (base, top, and height dimensions are 406, 126, and $140 \mathrm{~nm}$, respectively) is used for dimension normalization. Dozens of devices were tested, and NWs of similar base widths showed comparable device performance. The dc characteristics of the [00-1] NW MESFET on (110) substrates 
(a)
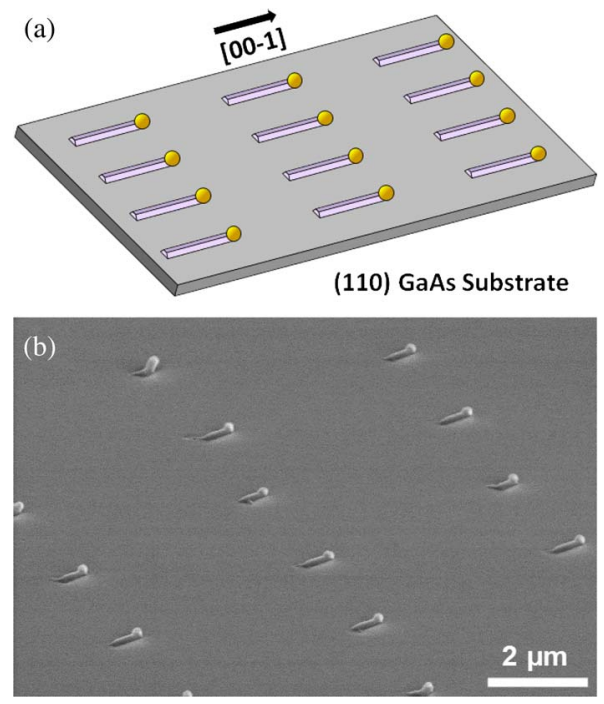

Fig. 3. (a) Illustration of site-controlled unidirectional planar NW array growth, with Au particles defined lithographically and growth taking place on a (110) substrate along [00-1] direction. (b) Tilted $\left(75^{\circ}\right)$ SEM micrograph of a completely ordered array of [00-1] GaAs NWs grown from E-beam patterned Au catalyst nanoparticles on a (110) GaAs substrate. The Au nanoparticle size is approximately $175 \mathrm{~nm}$ in diameter, and the NWs are $\sim 1.2 \mu \mathrm{m}$ long.

demonstrated here are comparable to that of the control devices grown on (100) substrates, with slight enhancement as a result of improved fabrication. This confirms that [00-1] planar NWs grown on (110) substrates are just as viable as on (100) substrates for MESFET devices yet with better manufacturability for array-based channels as will be shown hereinafter.

The successful demonstration of high-quality unidirectional planar NW growth by using (110) substrates makes it possible to realize completely ordered NW arrays for array-based high-performance nanoelectronics. The ordering will be determined by the site control of the Au catalyst nanoparticles by lithographical patterning. One of the challenges of patterning is the introduction of organic contamination from the resist chemicals. NWs are known to be sensitive to contaminants during growth as the metallic nanoparticles can absorb precursors and impurities alike depending on their solid solubility. Impurities can dope the wire as well as cause abnormal growth to occur [14]. Planar NWs are extremely sensitive to impurities as they can not only infiltrate the catalyst nanoparticle but also contaminate the substrate surface that the epitaxial relationship of the entire NW sidewall is built on, preventing planar NW growth. Depending on the substrate treatment, we have observed NWs grown initially in the planar fashion but taking off from the surface to grow in out-of-plane mode soon after. Extensive substrate cleaning, including oxygen plasma and extended solvent soaks as described in the experimental section, is utilized to prevent NW growth deviating from the planar mode. Shown in Fig. 3 is an array of planar GaAs NWs on a (110) substrate successfully grown from Au catalyst nanoparticles patterned using electron beam lithography. It is evident that all the NWs unanimously propagated along the same direction, [00-1]. Devices using a completely ordered GaAs NW array will be reported separately.

\section{CONCLUSION}

In summary, we have demonstrated the complete alignment of Au-catalyzed VLS planar GaAs NWs along the [00-1] crystal orientation by growing on (110) instead of (100) substrates. The unidirectional [00-1] NW-based long-channel MESFETs show excellent dc characteristics. With the inherent 3-D cross section, precise alignment and controlled placement through monolithic bottom-up growth demonstrated here, planar III-V NW array-based transistors could become a viable candidate for high-performance manufacturable bottom-up grown transistor technology beyond the Si roadmap.

\section{ACKNOWLEDGMENT}

The authors would like to thank Prof. P. Evans of the University of Wisconsin-Madison for discussions.

\section{REFERENCES}

[1] J. Appenzeller, J. Knoch, M. T. Bjork, H. Riel, H. Schmid, and W. Riess, "Toward nanowire electronics," IEEE Trans. Electron Devices, vol. 55, no. 11, pp. 2827-2845, Nov. 2008.

[2] H. J. Joyce, Q. Gao, J. Wong-Leung, Y. Kim, H. H. Tan, and C. Jagadish, "Tailoring GaAs, InAs, and InGaAs nanowires for optoelectronic device applications," IEEE J. Sel. Topics Quantum Electron., vol. 17, no. 4, pp. 766-778, Jul./Aug. 2011.

[3] M. Li, R. B. Bhiladvala, T. J. Morrow, J. A. Sioss, K. K. Lew, J. M. Redwing, C. D. Keating, and T. S. Mayer, "Bottom-up assembly of large-area nanowire resonator arrays," Nature Nanotechnol., vol. 3, no. 2, pp. 88-92, Feb. 2008.

[4] C. M. Lieber, B. Z. Tian, T. Cohen-Karni, Q. Qing, X. J. Duan, and P. Xie, "Three-dimensional, flexible nanoscale field-effect transistors as localized bioprobes," Science, vol. 329, no. 5993, pp. 830-834, Aug. 2010.

[5] T. J. Morrow, M. Li, J. Kim, T. S. Mayer, and C. D. Keating, "Programmed assembly of DNA-coated nanowire devices," Science, vol. 323, no. 5912, p. 352, Jan. 2009.

[6] J. Johansson, L. S. Karlsson, C. P. Svensson, T. Martensson, B. A. Wacaser, K. Deppert, L. Samuelson, and W. Seifert, "Structural properties of $<111>$ B-oriented III-V nanowires," Nature Mater., vol. 5, no. 7, pp. 574-580, Jul. 2006.

[7] J. Johansson, L. S. Karlsson, K. A. Dick, J. Bolinsson, B. A. Wacaser, K. Deppert, and L. Samuelson, "Effects of supersaturation on the crystal structure of gold seeded III-V nanowires," Cryst. Growth Des., vol. 9, no. 2, pp. 766-773, 2009.

[8] Z. Fan, J. C. Ho, Z. A. Jacobson, R. Yerushalmi, R. L. Alley, H. Razavi, and A. Javey, "Wafer-scale assembly of highly ordered semiconductor nanowire arrays by contact printing," Nano Lett., vol. 8, no. 1, pp. 2025, Jan. 2008

[9] S. A. Fortuna, J. Wen, I. S. Chun, and X. Li, "Planar GaAs nanowires on GaAs (100) substrates: Self-aligned, nearly twin-defect free, and transferprintable," Nano Lett., vol. 8, no. 12, pp. 4421-4427, Dec. 2008.

[10] G. Zhang, K. Tateno, H. Gotoh, and H. Nakano, "Parallel-aligned GaAs nanowires with 110 orientation laterally grown on [311] B substrates via the gold-catalyzed vapor-liquid-solid mode," Nanotechnology, vol. 21, no. 9, p. 095607 , Mar. 2010.

[11] S. A. Fortuna and X. Li, "GaAs MESFET with a high-mobility selfassembled planar nanowire channel," IEEE Electron Device Lett., vol. 30, no. 6, pp. 593-595, Jun. 2009.

[12] X. Miao and X. Li, "Scalable monolithically grown AlGaAs-GaAs planar nanowire high-electron-mobility transistor," IEEE Electron Device Lett., vol. 32, no. 9, pp. 1227-1229, Sep. 2011.

[13] K. Hiruma, M. Yazawa, T. Katsuyama, K. Ogawa, K. Haraguchi, M. Koguchi, and H. Kakibayashi, "Growth and optical properties of nanometer-scale GaAs and InAs whiskers," J. Appl. Phys., vol. 77, no. 2, pp. 447-462, Jan. 1995.

[14] R. E. Algra, M. A. Verheijen, M. T. Borgstrom, L. F. Feiner, G. Immink, W. J. P. van Enckevort, E. Vlieg, and E. P. A. M. Bakkers, "Twinning superlattices in indium phosphide nanowires," Nature, vol. 456, no. 7220, pp. 369-372, Nov. 2008. 